MOGOLLÓN, E.M. et al. Resposta superovulatória em vacas e novilhas Holandêsas no Valle de Chiquinquira, Colômbia. PUBVET, Londrina, V. 5, N. 16, Ed. 163, Art. 1102, 2011.

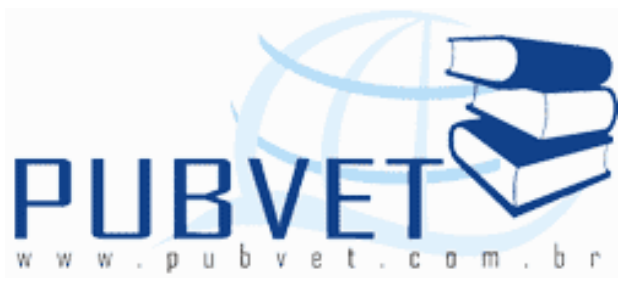

PUBVET, Publicações em Medicina Veterinária e Zootecnia.

\title{
Resposta superovulatória em vacas e novilhas Holandêsas no Valle de Chiquinquira, Colômbia
}

\author{
Mogollón, E. M. ${ }^{1}$, Jimenez, A. L. ${ }^{2}$ Cardona, J. R. ${ }^{3}$, Narvaez, H. J ${ }^{1}$; Quirino, C.R. ${ }^{4}$ \\ Beltrame, R.T. ${ }^{5}$
}

${ }^{1}$ Doutorando do Setor de Reprodução Animal, LRMGA/CCTA/UENF, Campos dos Goytacazes-RJ, Brasil. mao5_2000@yahoo.com

${ }^{2}$ Doutoranda do Setor de Sanidade Animal, LSA/CCTA/UENF, Campos dos Goytacazes-RJ.

${ }^{3}$ Profissional autônomo (Ganadería La Alameda, Chiquinquira- Colombia)

${ }^{4}$ Professora Associada do Laboratório de Reprodução e Melhoramento Genético Animal, CCTA- UENF.

${ }^{5}$ Doutor em Ciência Animal, Professor UNESC, Colatina. ES.

\section{Resumo}

Foram analisadas produções oriundas de 255 colheitas de embriões em vacas e novilhas Holandêsas durante os anos de 2001 à 2004. Os animais foram selecionados por registros de produção e/ou pureza racial, sendo superovulados com Pluset $\AA$ ou Foltropin $\AA$. A superovulação foi iniciada a partir do dia 9 após o cio natural (DO) utilizando doses decrescentes de FSH via intramuscular (IM), aplicadas a cada 12 horas durante 4 dias. No terceiro dia de superovulação (D12) a tarde aplicou se prostaglandina (Ilirenß). Três inseminações artificiais (IA) foram consideradas, recuperando-se os embriões 
MOGOLLÓN, E.M. et al. Resposta superovulatória em vacas e novilhas Holandêsas no Valle de Chiquinquira, Colômbia. PUBVET, Londrina, V. 5, N. 16, Ed. 163, Art. 1102, 2011.

sete dias após a primeira IA de forma não cirúrgica. Os resultados obtidos sugeriram que o fármaco de superovulação utilizado pode influenciar a qualidade embrionária obtida. Ainda, a utilização de pluset ${ }^{\circledR}$ permite a produção de um maior número de embriões congeláveis em novilhas e folltropin $®$ em vacas.

Palavras-Chave: Superovulação, gonadotrofinas, transferência de embriões.

\title{
Superovulatory response in Holstein cows and heifers in the Chiquinquira valley, Colombia
}

\begin{abstract}
We analized production originated from 255 embryo recovery Holstein heifers and cows Turing years 2001 to 2004. The animals were selected for production records and racial purity, and superovulated with Pluset or Foltropin. Superovulation was iniciated on day 9 after natural Oestrus (D0) using decresing doses of FSH intramuscularly (IM), applied every 12 hours for 4 days. On the third day of superovulation (D 12) was applied prostaglandin (Illiren) in the afternoon. Three artificial insemination (IA) were found and recovered the embryos seven days alter the first IA in a non-surgical process. The results suggested that the drug of superovulation used can influence the embryo quality obtained. The use of Plused enables production greater number of freezable embryos in heifers and Foltropin in the cows.
\end{abstract}

Keywords: Superovulation, gonadotrophins, embryo transfer.

\section{Introdução}

A produção comercial de embriões in vivo (TE) e in vitro (PIVE) normalmente encontra-se atrelada a dois problemas. Inicialmente, destaca-se uma grande variabilidade na resposta superovulatoria de fêmeas doadoras quando diferentes tratamentos superovulatorios são utilizados. Em adicional a logistica de compra e manutenção de fêmeas receptoras que serão utilizadas para a sincronização elevam os custos minimizando os resultados econômicos da atividade. Diante das diversas estratégias hoje existentes que se direcionam a 
MOGOLLÓN, E.M. et al. Resposta superovulatória em vacas e novilhas Holandêsas no Valle de Chiquinquira, Colômbia. PUBVET, Londrina, V. 5, N. 16, Ed. 163, Art. 1102, 2011.

redução de custos, a criopreservação permite congelar os embriões produzidos em um determinado momento, para serem transferidos em data futura (Hasler 2006; Jones et al 2008). Em estudos de simulação, Beltrame et al (2007) demonstraram que esta técnica têm efeito financeiro positivo.

O número de folículos recrutados por onda de crescimento folicular apresenta diferenças entre indivíduos. Embora estas diferenças possam ser decorrentes de diversos fatores, essa característica possui alta repetibilidade durante a vida reprodutiva da fêmea (Boni et al., 1997). Alguns estudos demonstram que a utilização de diferentes preparados comerciais de Hormônio Folículo Estimulante (FSH) podem influênciar o número de folículos recrutados (Referência). Comercialmente na Colômbia dois produtos estão disponíveis. No caso do Folltropin-V® uma constância de baixa proporção de FSH:LH é assegurada (Armstrong \& Opavsky, 1986; Lindsell et al., 1986). No Pluset a razão FSH/LH é elevada (1:1), mas segundo vários autores, o resultado de superovulação é melhor se comparado com os produtos de FSH com baixa concentração de LH (Lauria et al., 1983; Coulthard \& Burton, 1991; Cremonensi et al., 1992; Boland, 1995).

Diante do descrito, este estudo teve como intuito identificar o efeito de dois fármacos superovulatórios (Pluset ${ }^{\circledR}$ e Foltropin $\AA$ ) e da idade dos animais sobre o número de estruturas recuperadas no lavado, a qualidade embrionária, o estágio de desenvolvimento, e o número de estruturas passiveis de criopreservação (grau 1 e 2 IETS), produzidos por TE em uma propriedade na Colombia entre os anos 2001 e 2004.

\section{Material e Métodos}

O trabalho foi realizado na fazenda "La Alameda" no município de "San Miguel de Sema" (Boyacá- Colombia), pertencente aos denominados vales de "Ubate" e "Chiquinquirá". A precipitação media da região é de $1500 \mathrm{~mm} /$ ano, com temperatura media de $12,8{ }^{\circ} \mathrm{C}$ e $2550 \mathrm{~m}$ acima do nível do mar. 
MOGOLLÓN, E.M. et al. Resposta superovulatória em vacas e novilhas Holandêsas no Valle de Chiquinquira, Colômbia. PUBVET, Londrina, V. 5, N. 16, Ed. 163, Art. 1102, 2011.

Foram analisadas produções oriundas de 255 colheitas de embriões em vacas e novilhas Holandêsas durante os anos de 2001 à 2004. Os animais foram selecionados por registros de produção e/ou pureza racial, sendo superovulados com Pluset $\AA$ ou Foltropin $\AA$. As doses dos fármacos superovulatórios partiram de ensaios prévios realizados na mesma propriedade, os quais apresentaram resultados aceitáveis confirmados através de ultrasonografias ovarianas durante a execução (Singh, J. 2004). As doses totais de Pluset $\AA$ utilizadas foram de 380 UI e 365 UI, e Foltropin $®$ de 400 e $300 \mathrm{mg}$ para vacas e novilhas respectivamente.

A superovulação foi iniciada a partir do dia 9 após o cio natural (D0) utilizando doses decrescentes $(40 \%, 30 \%, 20 \%$ e 10\% - manhã e tarde) de FSH via intramuscular (IM), aplicadas a cada 12 horas durante 4 dias. No terceiro dia de superovulação (D12) a tarde aplicou-se $0,5 \mathrm{mg}$ de Cloprostenol, um análogo da prostaglandina F2a (Iliren $® 2 \mathrm{ml}$ intramuscular, Intervet, Colombia ) .

Três inseminações artificiais (IA) foram consideradas. A primeira IA foi realizada 12 horas após a detecção do estro que foi realizada de forma visual (6 da manhã e 6 da tarde) por aproximadamente 30 minutos. As outras inseminações seguiram-se entre 12 e 24 horas após a primeira IA.

A coleta dos embriões foi realizada sete dias depois da primeira inseminação. As fêmeas foram submetidas à anestesia epidural (4 ml de Lidocaína ${ }^{\circledR}$ 2\%) e foi realizado o lavado uterino mediante o uso de cateteres de "Foley". A lavagem foi realizada com PBS enriquecida com soro fetal bovino (Dulbecco'sPBS, Lab. Gibco, EUA) e utilizou-se um filtro, no final do sistema de condução, para manter os embriões na solução o tempo todo e recuperá-los por meio da lavagem da rede do filtro.

O líquido contido no filtro foi transferido para uma placa de Petri, onde se realizou a seleção e a classificação dos embriões.

Todos os lavados foram executados pelo mesmo técnico. As estruturas recuperadas foram contadas e classificadas segundo as normas da IETS procedendo-se o envase e congelamento das estruturas de qualidade 1 e 2 
MOGOLLÓN, E.M. et al. Resposta superovulatória em vacas e novilhas Holandêsas no Valle de Chiquinquira, Colômbia. PUBVET, Londrina, V. 5, N. 16, Ed. 163, Art. 1102, 2011.

(Leibo 1989, citado por Cabodevila 1993, Martins, C. 2007 ). Estruturas não fertilizadas foram designadas quando não havia sinal de divisão. (Olivera et al. 1993; Strigfellow, D. e Seidel, S. 1998; Machado-Pfeifer, L. et al. 2002).

Estatisticamente foi utilizada a análise de variância do número de estruturas congeladas, do número de mórulas, do número de blastocisto iniciais e do número de blastocistos para verificar se existiam diferenças entre os fármacos utilizados e as categorias de fêmeas doadoras. As médias destas características foram comparadas usando-se o Teste SNK (P>0,05)(SAS, 1999).

\section{Resultados}

Os resultados obtidos sugeriram que o número de embriões congeláveis foi superior para a categoria das vacas superovuladas por Foltropin $®$. Entretanto, para novilhas melhores resultados foram evidenciados com uso de Pluset ${ }^{\circledR}$ (Tabela 1). Quando comparados os tratamentos entre as categorias somente foram observadas diferenças mediante uso de Foltropin $®$.

Tabela 1. Medias e respectivos desvio padrão do número de estruturas congeladas para as categorias de doadoras vacas e novilhas, de acordo com o tratamento utilizado (Foltropin $₫$ - Pluset $\AA$ )

\begin{tabular}{|c|c|c|}
\hline Categoria & Novilha & Vaca \\
\hline \multicolumn{3}{|l|}{ Tratamento } \\
\hline FOLTROPIN $®$ & $6.82 \pm 3.68 \mathbf{B b}$ & $10.38 \pm 5.66 \mathbf{A a}$ \\
\hline PLUSET $®$ & $9.15 \pm 5.45 \mathbf{A a}$ & $7.93 \pm 3.45 \mathbf{B a}$ \\
\hline
\end{tabular}

Médias com letras em maiúsculas e diferentes na mesma coluna diferem pelo teste SNK ao $5 \%$

Médias com letras em minúsculas e diferentes na mesma linha diferem pelo teste SNK ao $5 \%$ 
MOGOLLÓN, E.M. et al. Resposta superovulatória em vacas e novilhas Holandêsas no Valle de Chiquinquira, Colômbia. PUBVET, Londrina, V. 5, N. 16, Ed. 163, Art. 1102, 2011.

$\mathrm{Na}$ avaliação da produção de mórulas, a utilização de Foltropin ${ }^{\circledR}$ em vacas obteve aproximadamente 6 embriões de media. Diferença foi apresentada entre os dois produtos comerciais em ambas as categorias. Em novilhas, considerando-se a produção de mórulas, um melhor desempenho foi obtido mediante utilização de Pluset $®$.

A média do número de blastocistos iniciais foi melhor na categoria vacas com Foltropin ${ }$, mas não apresentou diferença ao se comparar os dois produtos comerciais (Foltropin $®$ - Pluset $®$ ) dentro desta categoria. Para novilhas os dois produtos usados apresentaram diferenças sendo superiores os resultados com Pluset $®$ (tabela 2).

Tabela 2. Medias e respectivos desvio padrão do número de blastocistos iniciais para as categorias vaca e novilha, de acordo com o tratamento utilizado

\begin{tabular}{lll}
\hline Categoria & Novilha
\end{tabular}

Tratamento

\begin{tabular}{lll}
\hline FOLTROPIN $®$ & $3.27 \pm 2.26 \mathbf{B b}$ & $4.79 \pm 3.06 \mathbf{A a}$
\end{tabular}

PLUSET®

$4.20 \pm 2.84 \mathrm{Aa}$

$4.56 \pm 2.46 \mathbf{A a}$

(Foltropin $\AA$ - Pluset $\AA$ )

Médias com letras em maiúsculas e diferentes na mesma coluna diferem pelo teste SNK ao $5 \%$

Médias com letras em minúsculas e diferentes na mesma linha diferem pelo teste SNK ao $5 \%$

O número de blastocistos foi maior na categoria novilhas com o uso de

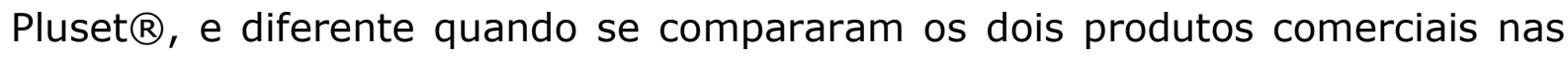
categorias (tabela 4.). O uso de Foltropin ${ }^{\circledR}$ não apresentou diferença entre as categorias, contrastando ao uso de Pluset $\AA$. 
MOGOLLÓN, E.M. et al. Resposta superovulatória em vacas e novilhas Holandêsas no Valle de Chiquinquira, Colômbia. PUBVET, Londrina, V. 5, N. 16, Ed. 163, Art. 1102, 2011.

Tabela 3. Medias e respectivos desvio padrão do número de Blastocistos para as categorias vaca e novilha, de acordo com o tratamento utilizado (Foltropin $₫$ - Pluset $($ )

\begin{tabular}{lll}
\hline Categoria Novilha & Vaca
\end{tabular}

Tratamento

\begin{tabular}{lll}
\hline FOLTROPIN $~$ & $5.42 \pm 2.85 \mathrm{Ba}$ & $4.87 \pm 2.75 \mathrm{Aa}$
\end{tabular}

\begin{tabular}{lll}
\hline PLUSET $尺$ & $7.05 \pm 6.61 \mathrm{Aa}$ & $3.70 \pm 2.00 \mathrm{Bb}$
\end{tabular}

Médias com letras em maiúsculas e diferentes na mesma coluna diferem pelo teste SNK ao $5 \%$

Médias com letras em minúsculas e diferentes na mesma linha diferem pelo teste SNK ao $5 \%$

\section{Discussão}

O hormônio $\mathrm{FSH}$, é produzido, armazenado e liberado pela hipófise anterior. É uma gonadotrofina que atua nos ovários estimulando o crescimento dos folículos e a secreção de estrógenos. Nos procedimentos de superovulação, geralmente é administrado em injeções múltiplas por possuir uma meia vida curta (Gonçalves et al, 2008).

Alguns estudos têm reportado resposta superior na produção de embriões com Pluset $\AA$. Tal fato tem sido atribuído as maiores concentrações de LH (Kelly 1997). Deve-se destacar que na raça Holandesa a expressão dos receptores de LH aumenta no momento da divergência folicular o que deveria ter favorecido o uso de Pluset que teoricamente tem um conteúdo mais alto de LH (Gimenes 2008).

Embora outros pesquisadores como Donaldson et al (1986) e Donaldson et al (1987) demonstraram menores taxas de ovulação e fecundação quando a porção de LH utilizada na superovulação foi excessiva, não entanto Alvarez et 
MOGOLLÓN, E.M. et al. Resposta superovulatória em vacas e novilhas Holandêsas no Valle de Chiquinquira, Colômbia. PUBVET, Londrina, V. 5, N. 16, Ed. 163, Art. 1102, 2011.

al (2006) não encontraram diferencia significativa entre Pluset e Foltropin utilizados por via subcutânea na superovulação de vacas da raça Caracu.

A idéia de que a resposta superovulatoria é afetada basicamente pelo estado dos ovários e pelo desenvolvimento folicular prévio ao inicio do tratamento parece ser seguida por alguns pesquisadores. Devido a isso, grandes esforços são disponibilizados para a obtenção de protocolos que permitam uma melhor sincronização da onda folicular emergente (Dong-Soo et al. 2007; Barros, C. 2004; Ávila. 2002; Mapletoft et al. 1999).

As variações existentes na produção embrionária também podem ser oriundas de outros fatores. Esses fatores podem ser individuais, um animal respondendo diferente de outro com o mesmo tratamento superovulatório, idade e histórico reprodutivo, ou inerentes ao ambiente, como época do ano, estresse, manejo nutricional e status ovariano no momento do tratamento (MAPLETOFT et al., 2003).

O uso de novilhas na superovulação pode estar correlacionado com um menor número de estruturas recuperadas. Excetuando-se o número de blastocistos, neste trabalho um número menor de estruturas foi obtido para todas as outras classificações embrionárias. Na raça Nelore, novilhas apresentam uma menor taxa de resposta ao tratamento com gonadotropinas. Estes pesquisadores afirmam que a idade das doadoras influi na resposta superovulatória, pois novilhas e vacas de primeira lactação também apresentaram menor número de ovócitos e embriões coletados que as vacas mais velhas, de quinta ou sexta lactação (Santiago et al, 2002) . Outro fator que interfere na produção embrionária é a superestimulação ovariana. Esta normalmente esta correlacionada a diminuição no número de estruturas recuperadas visto o aumento da dose de FSH em vacas mais novas e novilhas.

\section{Agradecimentos}

Os autores agradecem à empresa "ganaderia La Alameda" de Chiquinquirá (Boyacá) por permitir o uso de seus gados de produção para a realização deste projeto e particularmente ao senhor Anastasio Ortegón pelo interes no fomento das biotecnologias reprodutivas bovinas. 
MOGOLLÓN, E.M. et al. Resposta superovulatória em vacas e novilhas Holandêsas no Valle de Chiquinquira, Colômbia. PUBVET, Londrina, V. 5, N. 16, Ed. 163, Art. 1102, 2011.

\section{Referencias}

Acosta, D; Hernández, O. Evaluación de dos tratamientos de superovulación en vacas Brahman en un programa de transferencia de embriones. Tesis de grado Universidad de los Llanos. Escuela de Medicina Veterinaria y Zootecnia. Villavicencio. 2003.

Alvarez, R; Pires, R; Martinez, A. Resposta ovariana e produção de embriões de vacas superovuladas com Pluset ou Foltropin em dose única subcutânea. Acta Scientiae Veterinariae, v. 34 (suplemento 1) , p.516, 2006.

Ávila, J. Nuevas estrategias aplicadas a la superovulación y transferencia de embriones. En: Memorias IX curso internacional de reproducción Bovina. Universidad Autónoma de México. 2002.

Barros, C; Nogueira, M. Superovulação em zebuínos de corte. Em: $1^{\circ}$ Simpósio internacional de reprodução animal aplicada. Biotecnologia da reprodução em bovinos 2004.

Baruselli, P; Mucciolo, R; VIsintin, J; Viana, W; Arruda, R; Madureira,E e Molero- Filho, J. Transferência de embriões em bubalinos (Bubalus bubalis) Revista Zootecnia. 32, 1994.

Boni R, Roelofsen MWM, Pieterse MC, Kogut J, Kruip ThAM. Follicular dynamics, repeatability and predictability of follicular recruitment in cows undergoing repeated follicular puncture. Theriogenology, v.48, p.277-289, 1997.

Cabodevila, J. Superovulación En: Transferencia de embriones y biotecnología de la reproducción en la especie bovina. Ed. Acribia, Zaragoza. 1993.

DONALDSON, L.; WARD, D, ; GLENN, S. Use of porcine follicle stimulating hormone after chromatographic purification in superovulation of cattle. Theriogenology. v. 25, p. 747-757, 1986.

DONALDSON, L.; WARD, D. LH effects on superovulation and fertilization rates. Theriogenology. v. 27, p. 225, 1987.

Dong-Soo, S; Chang-Yong, C; Sun-Ho, C; Sang-Era, C; Hyun-Jong, K; Man-Hye,H; Il-Sun, R; Guk-Hyun, S; Ui-Hyung, K; Ill-Hwa,K. Effect of estradiol benzoate or GnRH treatment prior to superstimulation in CIDR-treated, Korean native cows (Bos taurus). Animal reproduction Science 100, 14- 21, 2007.

Gimenes, L; Sá Filho, M; Torres-Junior, J; Beltran, M; Nogueira, G.; Baruselli, P. Perfil de FSH e LH na divergência folicular em novilhas Nelore (Bos indicus). Braz. J. vet. Res. Anim. Sci., Sao Paulo, v. 45, p. 11- 16, 2008.

Hasler, J. Factors affecting frozen and fresh embryo transfer pregnancy rates in cattle. Theriogenology, v.56, p.1401- 1415, 2001.

Hasler, J. The Holstein cow in embryo transfer today as compared to 20 years ago. Theriogenology, v. 65, p. 4- 16, 2006.

Jones, A; Lamb, G. Nutrition, synchronization, and management of beef embryo transfer recipients. Theriogenology, v. 69 , p. 107-115, 2008.

Kelly, P; Duffy, P; Roche, J; Boland, M. Superovulation in cattle: effect of FSH type and method of administration of follicular growth, ovulatory response and endocrine patterns. Animal reproduction science, v. 46. p. 1- 14, 1997.

Leoni, G.; Bogliolo, L.; Berlinguer, F.; Rosati, I.; Pintus, P.; Ledda, S.; Naitana, S.; Defined media for vitrication, warming, and rehydratation: effects on post; thaw protein synthesis and viability of in vitro derived ovine embryos. Gryobiology, v.45, n. 3, p. 204; 212, 2002. 
MOGOLLÓN, E.M. et al. Resposta superovulatória em vacas e novilhas Holandêsas no Valle de Chiquinquira, Colômbia. PUBVET, Londrina, V. 5, N. 16, Ed. 163, Art. 1102, 2011.

Machado-Pfeifer, L; Correa, L; Pineschi, L. Alternativas hormonais para transferência de embriões em bovinos. Monografia Universidad Federal de Pelotas. Disponível na internet: www.ufsm.br/.../Aula\%20biotecnicas\%20ALTERNATIVAS\%20HORMONAIS\%20

Mapletoft, R; Martinez, M; Adams, G; Kastelic, J e Burnley, C. The effect of estradiol preparation on follicular wave emergence and superovulatory response in Norgestomet implanted cattle. Theriogenology, v. 51, p.411, 1999.

Mapletoft, R; Bennett, K; Adams, G. Recent advances in the superovulation in cattle. Rerpoduction and nutrition development, v. 42, p. 601-611, 2002.

Martins, C. Diferentes protocolos de superovulação com inseminação artificial em tempo fixo em Bos taurus e Bos indicus. São Paulo, 2007. 115f. Dissertação (mestrado) - Faculdade de Medicina Veterinária e Zootecnia, Universidade de São Paulo, São Paulo, 2007.

Mizuta, K. Estudo comparativo dos aspectos comportamentais do estro e dos teores plasmáticos de $\mathrm{LH}, \mathrm{FSH}$, progesterona e estradiol que precedem a ovulação em fêmeas bovinas Nelore (Bos taurus indicus), Angus (Bos taurus taurus) e Nelore x Angus (Bos taurus indicus x Bos taurus taurus). São Paulo, 2003. 98f. Tese (Doutorado) - Faculdade de Medicina Veterinária e Zootecnia, Universidade de São Paulo, São Paulo, 2003.

Olivera, M.; Martínez, N e Vélez, G. Importancia de las líneas genéticas, peso al nacimiento y destetes en un programa de transferencia de embriones. Revista El Cebú, n. 272, 1993

Singh, J; Dominguez, M; Jaiswal, R; Adams, G. Asimple ultrasound test to predict the superstimulatory response in cattle. Theriogenology. v. 62, p. 227; 243, 2004.

Strigfellow, D. e Seidel, S. Manual of the embryo transfer society. Tercera edición 1998

Serrato, J e Vargas, O. Utilización de la solución Hartman como medio para el lavado y transferencia de embriones bovinos. Tesis de grado Universidad de los Llanos. Escuela de Medicina Veterinaria y Zootecnia. 1991 